\title{
Intra-Appendicular Metallic Foreign Body: Case Report and Review of Literature
}

Amine Hamdane ${ }^{1 *}$, Mohammed Amine Hamouchi ${ }^{1}$, Oleko Eddy ${ }^{1}$, Ouadii Mouaqit ${ }^{1}$, El Bachir Benjelloun ${ }^{1}$, Abdelmalek Ousadden $^{1}$, Khalid ait Taleb ${ }^{1}$, Hicham El Bouhaddouti ${ }^{1}$

${ }^{1}$ Faculty of Medicine and Pharmacy, Sidi Mohammed Ben Abdellah university of Fez, Morocco, Visceral Surgery department A, CHU
Hassan II, Fez, Morocco

DOI: $\underline{10.36347 / \text { sasjs.2021.v07i04.009 }}$

| Received: 26.02.2021 | Accepted: 05.04.2021 | Published: 18.04.2021

*Corresponding author: Amine Hamdane

\section{Abstract}

Intra-appendicular foreign bodies are rare incidences that can lead to appendicitis. We report the case of a 24 year old female patient who was diagnosed an appendicular syndrome one week after accidental ingestion of a pin, hence the indication for appendectomy.

Keywords: Intra-Appendicular, Metallic Foreign Body Literature.

Copyright $(\mathcal{C} 2021$ The Author(s): This is an open-access article distributed under the terms of the Creative Commons Attribution 4.0 International License (CC BY-NC 4.0) which permits unrestricted use, distribution, and reproduction in any medium for non-commercial use provided the original author and source are credited.

\section{INTRODUCTION}

Intra-appendicular foreign bodies are rare events [1], their prevalence being estimated at $0.0005 \%$ with an incidence of 5 cases per 10 million populations $[1,2,4]$. The first case in history was described in 1736 , when an 11-year-old child was found to have an appendicular perforation by a pin, leading to the first appendectomy in history by the British surgeon Claudius Amyand [5]. The diagnosis is often made in the context of accidental or deliberate ingestion of metallic foreign body when an appendicular syndrome is present and the image of foreign body persists in the right iliac fossa after ingestion.

\section{PATIENT AND OBSERVATION}

A 24 year old female patient who accidentally ingested a pin, the patient presented with right iliac fossa pain radiating to the hypogastrium one week after ingestion, which led her to consult the emergency room. The clinical examination on admission revealed a febrile patient at $38.5^{\circ} \mathrm{C}$ with tenderness in the right iliac fossa. Abdominal ultrasound suspected a foreign body in the right iliac fossa, abdominal CT scan showed a metallic foreign body intra-appendicular without radiological signs of peritonitis (Figure 1). The patient was admitted to the operating room, where surgical exploration revealed an inflamed appendix with an incarcerated pin within its lumen (Figure 2), with no intraperitoneal effusion or collection and no wound on the adjacent viscera. The procedure performed was an appendectomy (Figure 3), the postoperative course was uneventful.

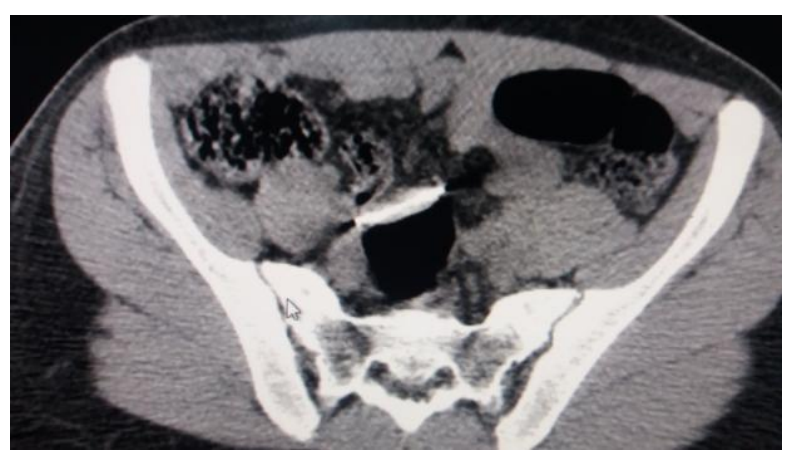

Fig-1: Abdominal CT scan. Presence of a metallic foreign body intra-appendicular without radiological signs of peritonitis

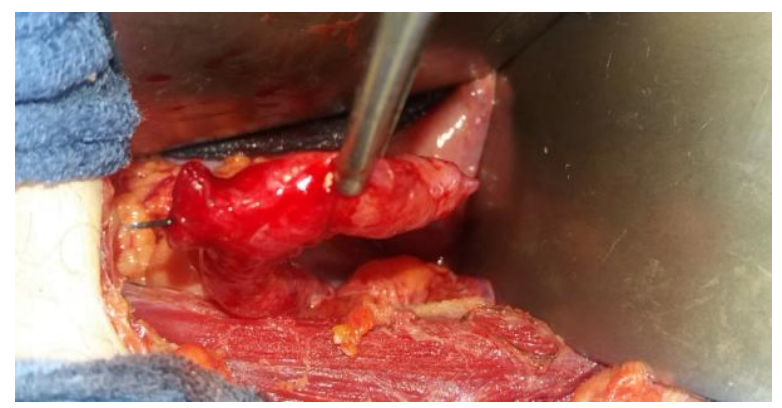

Fig-2: Inflamed appendix with an incarcerated pin within its lumen 


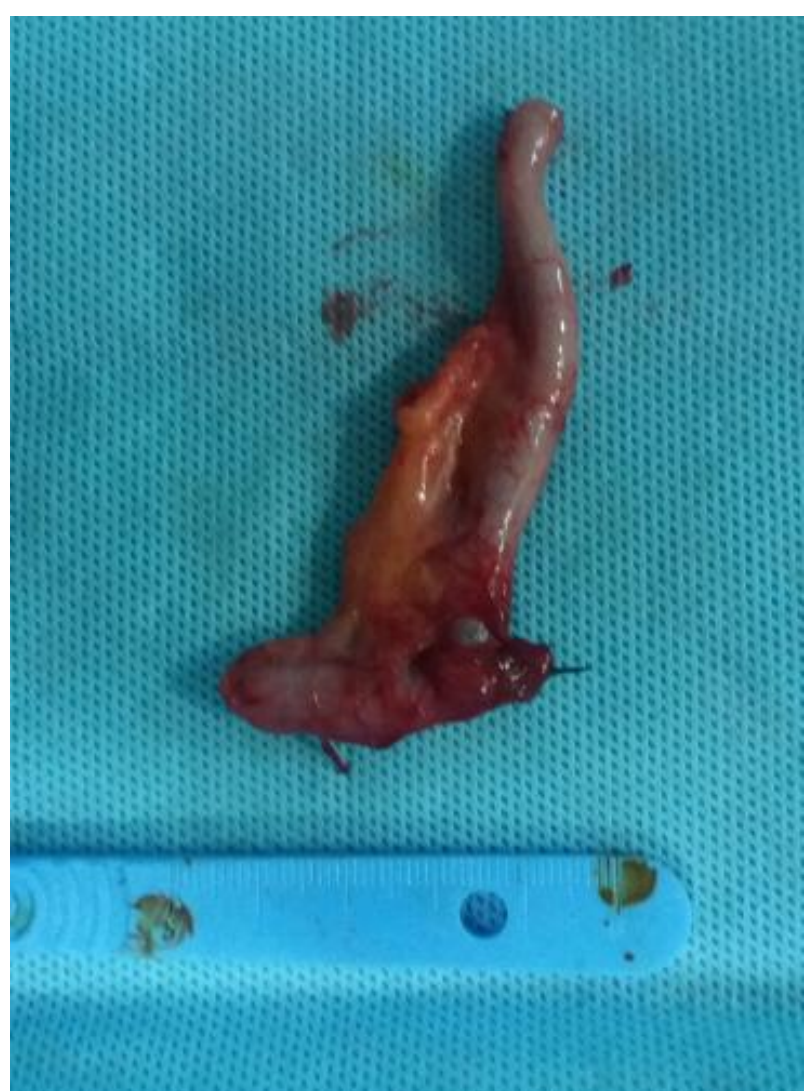

Fig-3: Surgical sample. Appendix with intra-luminal pin

\section{DISCUSSION}

Foreign body ingestion is a frequent entity, it is often observed in children [6, 7] as well as adults either accidentally or voluntarily especially in the context of psychiatric illness or particular contexts (psychomotor retardation, consumption of toxic substances, wearing of dental prostheses ...) [7, 8]. The presence of an intra-appendicular foreign body is a rare entity, 256 cases were reported in the 20th century [4], various objects were found; meat bones, needles, small stones, hair, nails, and even the tip of a thermometer $[2,3,9,11]$ as well as a few exceptional cases of foreign bodies of dental origin (prostheses, teeth, etc.) [1, 12, $13]$.

Digestive foreign bodies tend either to be eliminated spontaneously via the lower tract in the direction of peristalsis after a week and remain asymptomatic $[1,7,14]$ or to implant themselves at the level of physiological strictures [7, 14, 15]. Penetration of a foreign body into the appendix from the cecum depends on its weight; the diameter of the appendicular orifice as well as the anatomical position of the appendix, the retrocaecal appendix gives difficult access for foreign bodies [1, 2, 4]. Peristalsis is often insufficient to evacuate an intra-appendicular foreign body due to the formation of a faecal layer around the foreign body [4], and this may also delay the onset of an inflammatory reaction, which may take hours to months $[16,17]$. Rare cases of appendicitis have been described several months after ingestion $[10,18]$.
An intra-appendicular foreign body is manifested mainly by abdominal pain, particularly in the right iliac fossa, associated with vomiting and fever $[1,2,4,7]$. According to the literature, $70 \%$ of intraappendicular foreign bodies are symptomatic, with a clinical examination that may reveal tenderness in the right iliac fossa or even signs of localised or generalised peritoneal irritation $[4,7]$.

The management of an intra-appendicular foreign body depends on the clinical features and time of onset of symptoms. The most common complications are acute appendicitis as well as appendicular abscess which may result from appendiceal perforation. The frequency of these complications increases with sharp objects larger than $6 \mathrm{~cm}$ in length and/or $2.5 \mathrm{~cm}$ in diameter [7, 8, 15]. Endoscopic intervention may be suggested when possible. In case of failure or complication, appendectomy is indicated [4].

\section{CONCLUSION}

The intra-appendicular foreign body is a rare situation in our context, with frequent complications, and endoscopic extraction is proposed in the absence of complications. However, laparoscopic appendectomy remains the best therapeutic option.

\section{REFERENCES}

1. Truong Minh Khoa, Mathys Philippe Andreas, Petermann David. Intra-appendicular foreign body. Swiss Medical Forum. 2020;20(4546):655-657.

2. Balch CM, Silver D. Foreign bodies in the appendix. Report of eight cases and review of the literature. Arch Surg. 1971; 102(1):14-20.

3. Collins DC. 71,000 Human Appendix Specimens. A Final Report, Summarizing Forty Years' Study. Am J Proctol. 1963; 14:265-81.

4. Klingler PJ, Seelig MH, DeVault KR, Wetscher GJ, Floch NR, Branton SA. Ingested foreign bodies within the appendix: A 100-year review of the literature. Dig Dis. 1998; 16(5):308-14.

5. Amyand C. Of an inguinal rupture, with a pin in the appendix caeci incrusted with stone, and some observations on wound in the guts. Phil Trans R Soc Lond. 1736; 39:329-42.

6. Cheng W, Tam PK. Foreign-body ingestion in children: experience with 1,265 cases. J Pediatr Surg. 1999; 34(10):1472-6.

7. Birk M, Bauerfeind P, Deprez PH, Hafner M, Hartmann D, Hassan C. Removal of foreign bodies in the upper gastrointestinal tract in adults: European Society of Gastrointestinal Endoscopy (ESGE) Clinical Guideline. Endoscopy. 2016;48(5):489-96.

8. Committee ASoP, Ikenberry SO, Jue TL, Anderson MA, Appalaneni V, Banerjee S. Management of ingested foreign bodies and food impactions. Gastrointest Endosc. 2011;73(6):1085-91. 
9. Hazer B, Dandin O, Karakas DO. A rare cause of acute appendicitis: an ingested foreign body. Ulus Travma Acil Cerrahi Derg. 2013;19(6):570-2.

10. Antonacci N, Labombarda M, Ricci C, Buscemi S, Casadei R, Minni F. A bizarre foreign body in the appendix: A case report. World J Gastrointest Surg. 2013; 5(6):195-8.

11. Zardawi I, Siriweera E. Images in clinical medicine. Pellets in the appendix. N Engl J Med. 2013; 369(6):e7.

12. Mellor TK, Mellor SG. Foreign bodies of dental origin in the appendix. J R Army Med Corps. 1995; 141(3):174-6.

13. Tanaka K, Toyoda H, Aoki M, Noda T, Aota T. An incarcerated prosthetic tooth in the vermiform appendix. Gastrointest Endosc. 2007; 66(2):400-1; discussion 1.

14. Ginsberg GG. Management of ingested foreign objects and food bolus impactions. Gastrointest Endosc. 1995; 41(1):33-8.
15. Webb WA. Management of foreign bodies of the upper gastrointestinal tract: update. Gastrointest Endosc. 1995;41(1):39-51.

16. Pilichos C, Tasias G, Pyleris E, Anyfantis N, Pantelaros N, Barbatzas C. Endoscopic extraction of a metal key impacted within the appendix. World J Gastrointest Endosc. 2010;2(11):372-4.

17. Zampieri N, Zuin V, Ottolenghi A, Camoglio FS. Recurrent abdominal pain due to buckshots in the appendix. Acta Paediatr. 2008; 97(7):983-4.

18. Ozguner IF, Boduroglu E, Cavusoglu YH, Erdogan D. Appendicitis occurring 3 years after ingestion of metallic pin. Turk J Gastroenterol. 2012;23(2):1878.

19. Lam HC, Woo JK, van Hasselt CA. Management of ingested foreign bodies: a retrospective review of 5240 patients. J Laryngol Otol. 2001;115(12):954-7. 